\section{Evaluation of corpus geniculatum laterale and vitreous fluid by magnetic resonance spectroscopy in patients with glaucoma; a preliminary study}

S Doganay', C Cankaya and A Alkan²
${ }^{1}$ Department of Ophthalmology, Inonu University School of Medicine, Malatya, Turkey

${ }^{2}$ Department of Radiology, Bezmialem Vakif University School of Medicine, Istanbul, Turkey

Correspondence: S Doganay, Department of Ophthalmology, Inonu University School of Medicine, Turgut Ozal Medical Center, Malatya TR-44280, Turkey Tel: + 9042234106 60; Fax: + 904223410728 E-mail: sdoganay@ inonu.edu.tr

Received: 12 August 2011 Accepted in revised form: 28 January 2012 Published online: 25 May 2012

\begin{abstract}
Purpose To determine whether metabolite changes at cellular level occur in vitreous and lateral geniculate body (LGB) in patients with glaucoma by magnetic resonance spectroscopy (MRS).

Methods A total of 29 eyes of 29 patients with glaucoma, no existing ocular pathology and systemic disease (group 1), and 13 eyes of 13 healthy individuals whose routine ophthalmological examinations were normal (group 2) were included in the study. Singlevoxel MRS examination was performed by placing region of interest in vitreous and LGB. Glutamate-glutamine (Glx)/creatin (Cr) ratios and lactate peaks in the vitreous, and the Glx/Cr, $\mathrm{N}$-acetyl aspartate (NAA)/Cr, choline $(\mathrm{Cho}) / \mathrm{Cr}$ ratios in the ipsilateral area of LGB were evaluated.

Results A statistically significant difference was found between the two groups for the $\mathrm{Glx} / \mathrm{Cr}$ ratio in both the vitreous and LGB $(P=0.0001)$. There was no statistically significant difference between the two groups for the $\mathrm{NAA} / \mathrm{Cr}$, and $\mathrm{Cho} / \mathrm{Cr}$ ratios in the LGB $(P=0.108)$. A lactate peak was established in the vitreous of $\mathbf{1 1}$ glaucoma patients.

Conclusion Determining increased Glx/Cr ratios in both the vitreous and LGB of glaucoma patients, supports the theory of apoptosis in the etiopathogenesis of glaucoma. The MRS method, which can measure biochemical structures and metabolites of tissues, and also shows on a single spectrum, may be a new and noninvasive method for confirming the diagnosis
\end{abstract}

of glaucoma and the role of apoptosis in the etiopathogenesis of glaucoma.

Eye (2012) 26, 1044-1051; doi:10.1038/eye.2012.84; published online 25 May 2012

Keywords: glaucoma; glutamate; MR spectroscopy; apoptosis

Introduction

Glaucoma is a specific optic neuropathy involving progressive damage to the optic nerve, deterioration of the retinal ganglion cells, and ultimately visual field loss. It is a leading cause of blindness worldwide, and is also described as one of the preventable causes of blindness if diagnosed early. ${ }^{1}$ The progressive damage in the visual fields of some patients with normal or controlled intraocular pressure (IOP) proves that the IOP is not only the cause of glaucoma, but is also an important risk factor for it. ${ }^{2}$

The importance of both the anterograde and retrograde neurotransmitter passage between the axons of the lateral geniculate body (LGB) and the retinal ganglion cells in maintaining the vital functions of the retinal ganglion cells is well known. It is thought that this passage is impeded reciprocally, especially in the intraocular part of the optic nerve. The effects of various growth factors, especially the brainderived neurotrophic factor on ganglion cells have been studied, and reported that these factors are produced in the superior colliculus or geniculate nucleus in the brain, then move retrogradely to the soma and work there to maintain the cell functions. ${ }^{3}$ 
Glutamate is an important transmitter existing widely in the central nervous system (CNS) and is responsible for excitatory transmission. ${ }^{1,4-6}$ Its neurotoxic effect (excitotoxicity) was first seen through experimental studies on mice. ${ }^{4}$ In further studies, it was determined that glutamate may be effective in the pathogenesis of some neurodegenerative diseases of CNS. ${ }^{4}$ In the light of the apoptosis theory indicated as a factor in glaucoma pathophysiology, an increase in the level of glutamate known for its neurotoxic effects, is expected on the optic disc vitreous and LGB region. Research carried out on glaucoma patients showed discrepancies in their glutamate levels in the vitreous. ${ }^{7}$ Some studies reported an increase in the glutamate level, ${ }^{7}$ while others claimed no increase in glutamate. ${ }^{1}$ However, neuroexcitoxicity was shown histopathologically in the experimental glaucoma models. ${ }^{5}$ Thus, recent studies have mainly concentrated on searching for the remediation role of antiexcitoxic agents, such as memantine, and most of these studies have revealed that memantine is an effective agent for the treatment of experimental glaucoma. 8,9 In relation to this topic, an unmasked, phase III clinical trial, examining the safety and efficacy of oral memantine as a neuroprotectan for the treatment of glaucoma was announced a few years ago. The study showed that there was no statistical difference about the progression of the disease between the patients receiving memantine and placebo. As a consequence, there was no basis for the approval of memantine for use in glaucoma. ${ }^{10}$ But perhaps, with better parameters to define more precise endpoints and/or an extension of the trial, the efficacy of memantine might have achieved statistical significance as discussed in Osborne's article. ${ }^{11}$

Glaucoma is diagnosed when the characteristic optic disc changes are seen along with corresponding visual field defects by structure and function correlation. Threshold standard achromatic perimetry (SAP) replaced kinetic perimetry as the 'gold standard' for documenting functional losses caused by glaucoma. And though SAP is an excellent modality for documenting moderate-toadvanced optic nerve head $(\mathrm{ONH})$ and retinal nerve fibre layer damage, it is widely accepted that by the time threshold SAP reveals the first functional defects, nearly $50 \%$ of optic nerve axons have been lost. Namely, when the glaucoma is diagnosed clinically, there has already been a considerable damage in retinal ganglion cells. Frequency doubling technology (FDT) perimetry and short-wavelength automated perimetry (SWAP) offer unique opportunities toward the goal of earlier detection and diagnosis. Both of these technologies have been evolving. Applying both FDT and SWAP can bolster the functional glaucoma testing. Today neither of these technologies is intended to replace the current gold standard (threshold SAP). When one or both of these technologies are applied as adjuncts' to threshold SAP, we will be able to detect damage earlier than previously possible. ${ }^{12,13}$ Currently, axonal cell loss may precede perimetric evidence of such cell loss by years. For this reason, addition to previous methods, novel diagnostic methods are still required to establish the neuronal damage at cellular level.

Magnetic resonance spectroscopy (MRS) is a diagnostic method that is able to evaluate biochemical structure and metabolites of tissues non-invasively. ${ }^{14}$ In our study, we attempted to determine whether metabolite changes in cellular level occur in both vitreous and LGB of patients with glaucoma.

\section{Materials and methods}

In this study, 29 eyes of 29 glaucoma patients, suitable for criteria for inclusion, and 13 eyes of 13 healthy individuals, found normal in routine ocular examination, were included in the study. All the patients were enrolled from a single centre. The race of the participants was Caucasian. The patients with glaucoma were classified as group 1, and the healthy individuals were defined as group 2. The total study period was 6 months.

Patient inclusion criteria for group 1 were characterized by the presence of glaucomatous optic disc damage (glaucomatous cupping and/or glaucomatous $\mathrm{ONH}$ changes), and the presence of glaucomatous visual field defects detected in the computerized visual field examination. Individuals in group 2 were characterized by IOP $<21 \mathrm{~mm} \mathrm{Hg}$, normal optic disc, and normal visual field examination.

The visual field examination was performed using Humphrey (Carl Zeiss Meditec, Dublin, CA, USA) (30-2) SITA-Fast (Swedish Interactive Thresholding Algorithm) programme. The test was repeated under certain circumstances such as low reliability indices (fixation loss $>20 \%$ and false positive, and false negatives $>33 \%$ ) and poor cooperation. Visual field deficits in group 1 were categorized according to Hodapp-Parrish-Anderson classification.

Institutional Review Board approval was obtained from the Human Research Ethics Committee at the Inonu University School of Medicine. Informed consent was obtained from all of the subjects, and the study was conducted with the ethical standards outlined in the Declaration of Helsinki.

Exclusion criteria included patients with at least one of the following conditions; previous ocular surgery, laser therapy and trauma history, corneal and lens pathologies preventing ocular fundus examination, uveitis, and posterior segment pathologies. The glaucoma patients in group 1 were consulted to Neurology department. After consultation, the patients who had neurodegenerative 
diseases of CNS and had systemic diseases such as diabetes and hypertension were also excluded. A total of 11 patients were excluded; 8 were excluded before the MRS examination because of diabetes (4 patients) and hypertension (4 patients). A further three patients were excluded during the MRS examination because of CNS pathologies (two with suspected Alzheimer's disease, and one with suspected multiple sclerosis).

The glaucoma patients in group 1 had topical therapy for glaucoma and their IOP values were underpressure with topical antiglaucomatous therapy.

In all cases, the following tests were performed: visual acuity tests with and without corrective lenses, IOP by applanation tonometry, central corneal thickness measurement by using the Pentacam-Scheimpflug method, visual field examination using the (30-2) SITAFast programme (Humphrey, Carl Zeiss Meditec), a complete slit-lamp examination including gonioscopy, anterior segment examination, and a detailed fundus and optic disc examination by dilating the pupils.

\section{MRS technique}

After classifying the patients as group 1 and group 2, they were subjected to an MRS examination at the department of Radiology. All of the patients were warned not to move their eyes during the examination. Before the MRS, routine conventional magnetic resonance imaging (MRI) sections were obtained using MRI equipment (Gyroscan Intera Master, Philips, Best, Holland, 1.5 T, 0.33mT). In the examination, axial plane T1A T1A (TR450 ms, TE: $10 \mathrm{~ms}$ ), axial T2 A (TR $4366 \mathrm{~ms}$, TE: $120 \mathrm{~ms}$ ), coronal FLAIR (TR6000 ms, TE: $110 \mathrm{~ms}$, TI: 2000), and sagittal T2 A
(TR 4366 ms, TE: $120 \mathrm{~ms}$ ) views were obtained. The section thickness and number of slice acquisition were maintained constant at $4 \mathrm{~mm}$ and $2 \mathrm{~mm}$, respectively. The patients who had CNS pathologies detected as a result of the examination were excluded from the study. A single-voxel MRS examination was performed by placing region of interest (ROI) into the corpus vitreous and LGB on the same side. When placing the ROI, any contact with bone, vessels, air, and fat was avoided. A $13 \times 13 \mathrm{~mm}$ (TE: $31 \mathrm{~ms}$ ) ROI in vitreous fluid and a $12 \times 12 \mathrm{~mm}$ (TE: $136 \mathrm{~ms}) \mathrm{ROI}$ in LGB were placed using the point resolved spectroscopy) technique. The ROI was placed by localizing the LGB anatomically with three plain, thin section MRI views. Before obtaining the spectrum, shimming was carried out to achieve field homogeneity, followed by water suppression, using a chemical-shift-selective saturation pulse. A spectral scan interval of $1000 \mathrm{~Hz}$ and 1024 pixel data size were used. After obtaining all the data, decoding and an elaboration of the data were performed using the software provided by the company. After obtaining the spectral pattern, baseline and phase correction processes were applied in all cases for which corrections are needed.

Glutamate-glutamine (Glx)/creatin $(\mathrm{Cr})$ ratios

(Figure 1) and lactate peaks (Figure 2) in vitreous in each case, and Glx/Cr, $\mathrm{N}$-acetyl aspartate (NAA)/Cr and choline (Cho)/Cr ratios in the ipsilateral area of LGB were evaluated by the MRS method and the results were exhibited in Figure 3.

\section{Assessment of the data}

After the completion of the MRI process, the spectrum was evaluated by a radiologist based on the agreement

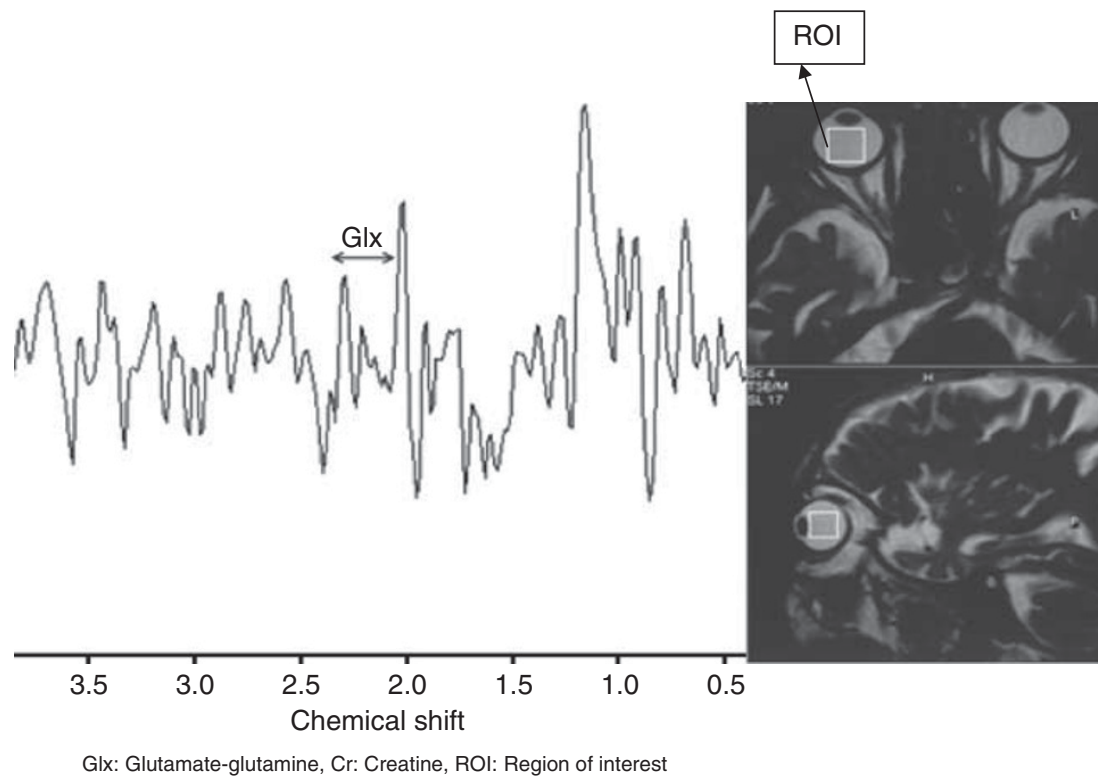

Figure 1 Increased Glx/Cr ratio that was detected between 2.1 and 2.5 p.p.m. in a glaucoma patient's vitreous by MRS. 


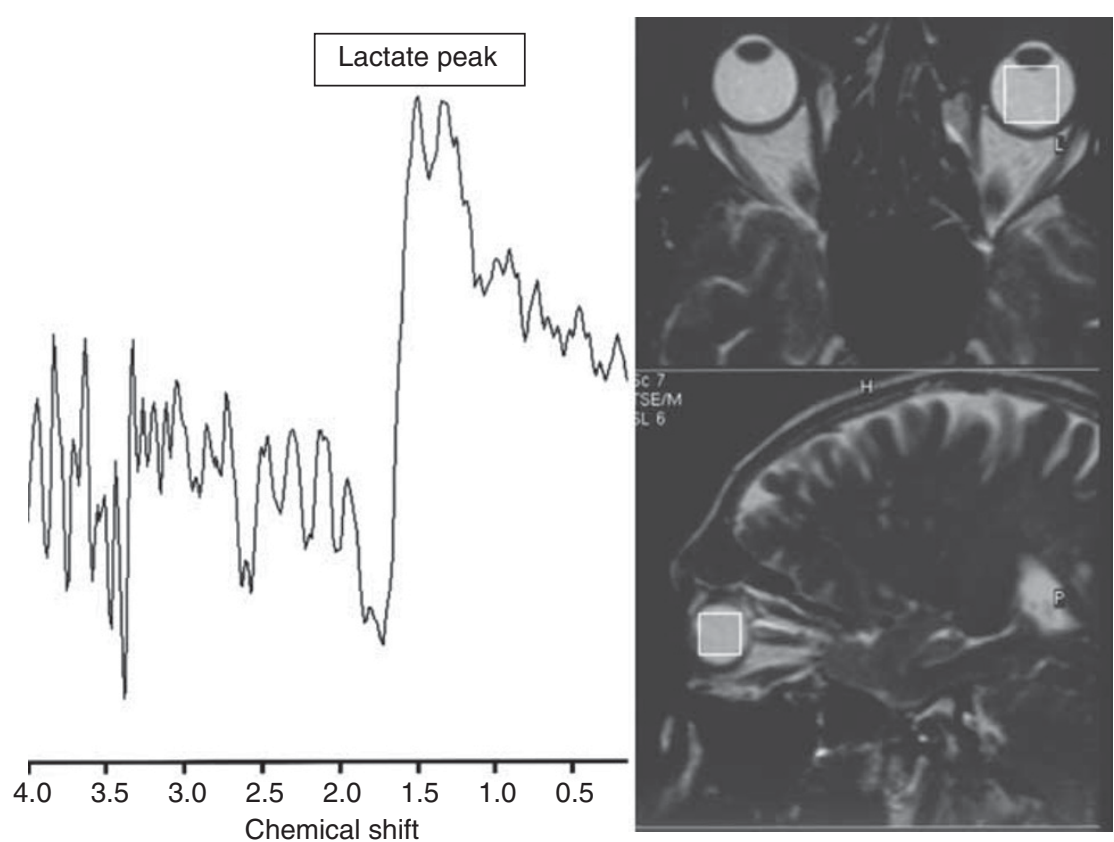

Figure 2 A lactate peak that was detected at 1.32 p.p.m. in left vitreous of a patient with glaucoma by MRS.

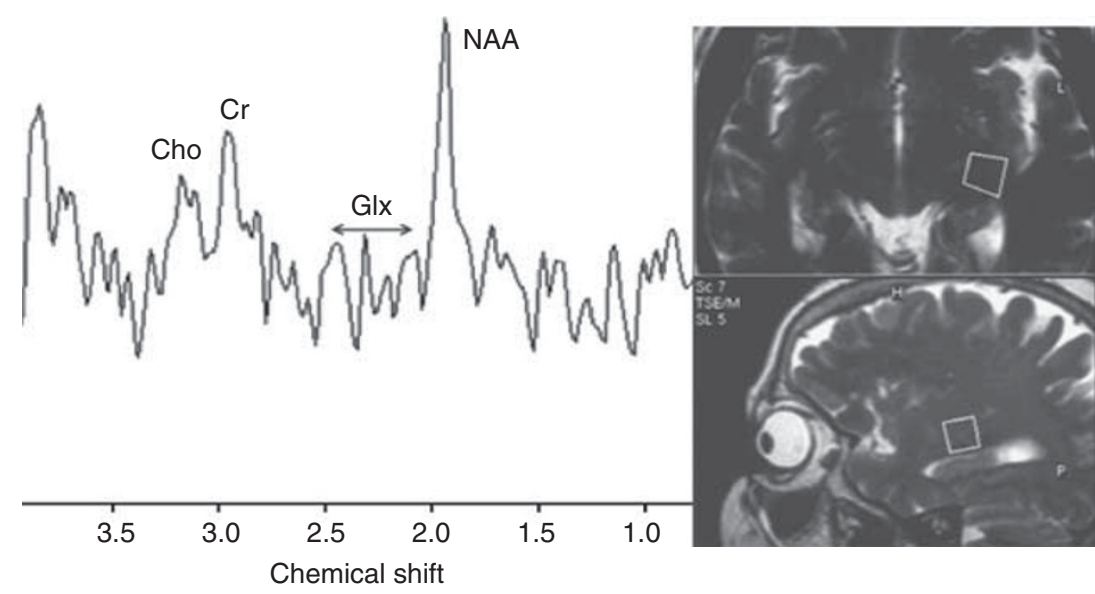

Cho: Choline, Cr: Creatine, Glx: Glutamate-Glutamine, NAA: N-Acetylaspartate

Figure 3 NAA peaks were detected at 2.0 p.p.m., Glx peaks were detected at $2.1-2.5$ p.p.m., Cr peaks were detected at 3.02 p.p.m., and Cho peaks were detected at 3.2 p.p.m. in the LGB region of a patient with glaucoma (group 1) by MRS.

principle. Resonance peaks in the spectrum was determined as NAA 2,0 p.p.m., Cr 3,02 p.p.m., Cho 3,2 p.p.m., Glx 2,1-2,5 p.p.m., lactate 1,3 p.p.m.. The area under the peaks was measured automatically by marking the lower borders of the peaks. These values were placed on an Excel table and NAA/Cr, $\mathrm{Cho} / \mathrm{Cr}$, and $\mathrm{Glx} / \mathrm{Cr}$ ratios were calculated.

\section{Statistical assessment}

For the statistical evaluation of the data of our study, SPSS for Windows Version 11.0 (SPSS Inc., Chicago, IL, USA) software was used. Measurable data of our study were presented as mean $\pm \mathrm{SD}$. The data did not conform to the normality assumption as evidenced by the 
Shapiro-Wilk test, $(P>0.05)$. Therefore, the MannWhitney $U$-test with the Bonferroni correction was used for the comparison of the groups. The $P$-value of $<0.05$ was taken to be statistically significant.

\section{Results}

The mean age was $65.8 \pm 5.4$ years in group 1 , and $62.8 \pm 4.2$ years in group 2 . No statistically significant difference in age $(P=0.064)$ and gender (in group 1 : 13 female and 16 male patients, in group 2: 7 female and 6 male individuals $(P=0.588)$ ) was found between the groups.

The mean value of the best corrected visual acuity was $0.3 \pm 0.2$ (logMAR) in group 1 , and $0.0(\log M A R)$ in group 2 $(P=0.001)$. The mean IOP value was $16.0 \pm 1.8 \mathrm{~mm} \mathrm{Hg}$ in group 1 and $13.6 \pm 2.4 \mathrm{~mm} \mathrm{Hg}$ in group $2(P=0.003)$. In group 1 , all the patients were diagnosed with primary open-angle glaucoma (POAG) by the gonioscopic examination. In group 1, 11 patients were receiving both prostaglandin analogue and brimonidine, 10 patients were receiving prostaglandin analogue monotherapy, 5 patients were receiving both prostaglandin analogue and dorzolamid/timolol maleat fixed combination, 2 patients were receiving a combination of prostaglandin analogue, dorzolamid/timolol fixed combination and brimonidine, and only 1 patient was receiving the dorzolamid/timolol maleat fixed combination. The mean duration time of the

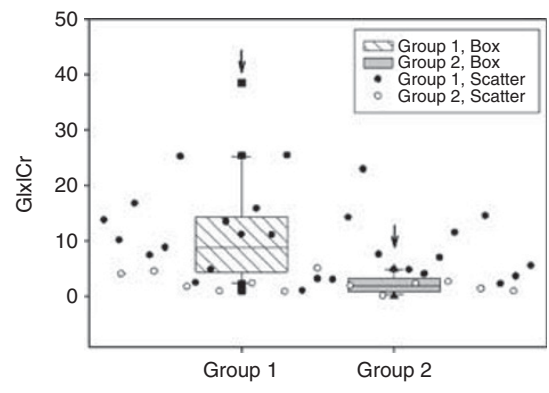

$\downarrow: p<0.05$, Glx: Glutamate-glutamine, Cr: Creatine, Group 1: Patients with glaucoma, Group 2: Healthy individuals

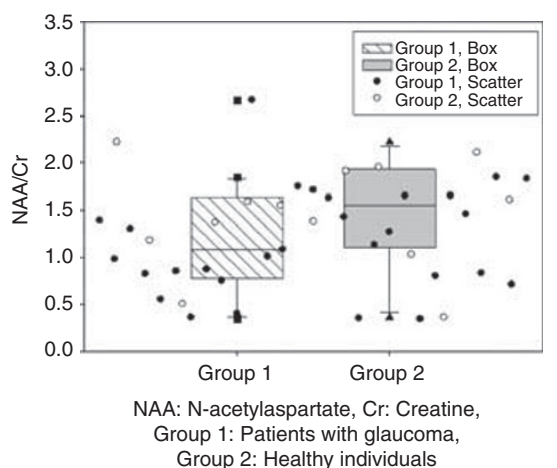

treatment was $7.7 \pm 2.8$ months in group 1 . When the visual field defects in the patients belonging to group 1 were categorized according to Hodapp-ParrishAnderson classification, 2 of them had early visual field loss, 18 of them had moderate visual field loss, and 9 of them had severe visual field loss. The mean cup-to-disc ratio was $0.7 \pm 0.1$ in group 1 .

In the glaucoma group (group 1), the mean $\mathrm{Glx} / \mathrm{Cr}$ value was $10.87 \pm 8.58$ in the vitreous, and the mean Glx/ $\mathrm{Cr}, \mathrm{NAA} / \mathrm{Cr}$ and $\mathrm{Cho} / \mathrm{Cr}$ values were $1.29 \pm 1.21$, $1.15 \pm 0.56,1.36 \pm 1.30$, respectively, in the LGB region. The mean Glx/Cr value was $2.24 \pm 1.51$ in the vitreous, and the mean $\mathrm{Glx} / \mathrm{Cr}, \mathrm{NAA} / \mathrm{Cr}$, and $\mathrm{Cho} / \mathrm{Cr}$ values were $0.38 \pm 0.35,1.44 \pm 0.57,0.79 \pm 0.32$, respectively, in the LGB region of healthy individuals (group 2). A lactate peak was established in the vitreous of 11 glaucoma patients in group 1 (Figure 2). A statistically significant difference was found between the two groups for $\mathrm{Glx} / \mathrm{Cr}$ values in both vitreous and LGB regions $(P=0.0001$;

Figure 4). There was no statistically significant difference between their NAA/Cr (Figure 4) and Cho/Cr values in LGB regions $(P=0.108$; Figure 4$)$.

\section{Discussion}

The Apoptosis theory in glaucoma was first described by Kerr and Wyllie in 1972. ${ }^{15}$ Apoptosis, in contrast to necrosis, is a cell death programmed genetically and

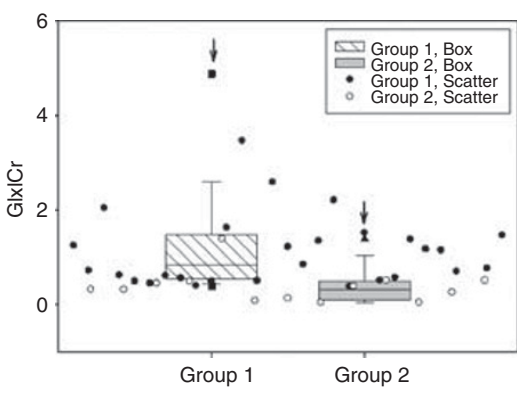

$\downarrow: p<0.05$, Glx: Glutamate-glutamine, Cr: Creatine, Group 1: Patients with glaucoma Group 2: Healthy individuals

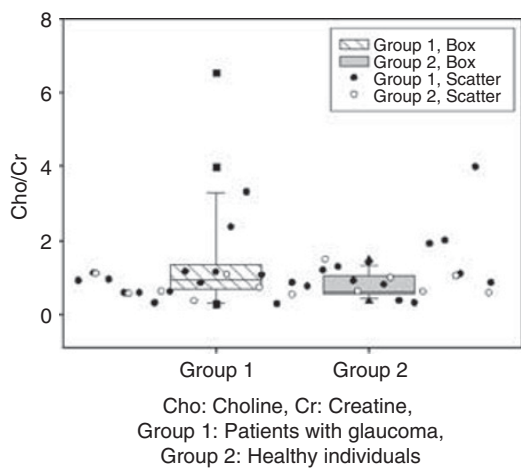

Figure $4 \mathrm{Glx} / \mathrm{Cr}$ ratios, NAA/Cr ratios, and $\mathrm{Cho} / \mathrm{Cr}$ in group 1 and group 2. 
shows no inflammation. With the effect of the endonucleases generated intracellularly, the cell kills itself by destroying its DNA and is phagocytized by the adjacent cells. ${ }^{16,17}$ Glutamate is a transmitter that exists widely in the brain and medulla spinalis, and is responsible for $75 \%$ of excitatory transmission. ${ }^{1,4-6}$ Glutamate triggers oxidative injury and free radical injury. It is also able to stimulate nitric oxide formation, which is an important free radical precursor, by activating nitric oxide synthase (iNOS or NOS-2) enzyme. ${ }^{18}$ Nitric oxide is a neurotransmitter and is involved in many pathological events. An increased nitric oxide activity in the $\mathrm{ONH}$ of the patients with open-angle glaucoma was reported. ${ }^{19}$ Free radicals are also formed in normal oxidative phosphorylation steps, as well as in glutamate receptor activation. Radicals, especially formed in very metabolically active tissues such as the retina, are inactivated by various enzymes. Retinal ganglion cell death is clinically known as optic neuropathy, whose most frequently encountered form is glaucoma. It has been studied on many antiapoptotic and neuroprotective mechanisms in glaucoma treatment. ${ }^{20}$ The first time in 1957; ganglion cell loss was shown in internal retinal layers after subcutaneous administration of glutamate to newborn mice. ${ }^{4}$ Subsequent studies showed that glutamate could also be effective in the pathogenesis of neurodegenerative diseases. ${ }^{4}$ However, antiexcitoxic therapy has only recently been introduced into the treatment of certain chronic neurological disorders. ${ }^{4}$

The growth factor for ganglion cells is the neurotrophic factor. According to novel theories, the blockage of the neurotrophic factor initiates apoptosis. Therefore, it is argued that the neuroprotective agents can be used in the therapy of glaucoma. A study, conducted by Yucel et $a l^{21}$ on nine monkeys with experimentally created glaucoma, showed that the use of memantine, which is a NMDA receptor blocker, gave better statistically significant results when compared with controls in neuronal damage and neuron atrophy in the LGB region.

In 1994, MRS was introduced to use in the area of ophthalmology by Berkowitz et al ${ }^{22}$ by investigating the lactate concentrations in rabbit vitreous. Subsequently, Rucker $e a^{23}$ analysed lactate concentration using MRS in the vitreous of four healthy individuals, and one patient with optic neuropathy. This study was the first to apply the MRS method to the human eye for the evaluation of the retina and optic nerve metabolism in healthy individuals.

In our study, we investigated the changes in Glx that is known for its neurotoxic effects in the vitreous and LGB regions of the patients with glaucoma using MRS imaging. There were two limitations that need to be acknowledged and addressed regarding this study. The first and the main limitation was related to non-consecutive patient selection owing to the MRS technique that requires high-tech, high cost as well as patient compliance. The second limitation was related to incompatibility of the patients to the visual field examination (SITA-Fast strategy) in particular patients with severe glaucomatous defects.

In the light of apoptosis theory, it can be expected that the level of glutamate, which is known for its neurotoxic effects, may lead to an increase in the $\mathrm{ONH}$, vitreous, and LGB. Honkanen et al ${ }^{1}$ compared glutamate and other amino-acid values in the vitreous of glaucoma patients undergoing a vitrectomy with the amino-acid values to the vitreous of the patients undergoing a vitrectomy for various reasons other than glaucoma. They did not determine any significant changes between the glutamate and other amino-acid values of the two groups. In some other studies, an increase of glutamate was found in the vitreous fluids in the glaucoma patients undergoing a pars plana vitrectomy, ${ }^{7}$ whereas some studies claimed that glutamate increases were not observed. ${ }^{1}$ In the study conducted by Honkanen et al, patients included in the study were not a homogenous group. Although only three of the total eight patients had POAG, others had a different type of glaucoma (two of them had primer angle closure glaucoma (PACG), two of them had uveitis with secondary PACG, and one of them had AxenfeldRieger syndrome). And also these populations were undergoing surgery because of various reasons, including epiretinal membrane, retinal detachment, aqueous misdirection, and uveitis. We think that these two distinct points were the possible reasons for the negative outcomes of Honkanen's study. However, neuroexcitoxicity shows histopathologically in the experimental glaucoma models and in the human trials with clinical glaucoma. It was found that glutamate levels in the vitreous and LGB region were comparable higher for the glaucoma group than the control group. These findings are in favour of the apoptosis theory charged in the etiopathogenesis of glaucoma.

The MRS provides information regarding the tissues, creating graphics with numeric values. Every peak in the spectrum is proportionate to the amount of chemical metabolites found; it is mainly based on determining the percentage of metabolite. The metabolites obtained in the spectrum are mainly NAA, Cr, Cho, and Glx. NAA is produced in neuronal mitochondria that move throughout the axon. It varies in proportion in different regions of the brain. Its proportion may be influenced by age and gender. It is accepted as a neuronal and axonal indicator. It has the largest peak in the adult brain spectrum. It provides data regarding the amount of live neurons in the area of examination. It is reduced in numerous pathologies that cause neuronal and axonal damage, such as a tumour, infarct, MS, and many 
leukoencephalopathies, epilepsy, and dementia. It does not exist outside of the CNS. ${ }^{24-26}$ In this study, no statistically significant difference was found, although $\mathrm{NAA} / \mathrm{Cr}$ ratios in the spectra obtained from the LGB in the patients with glaucoma were reduced when compared with healthy individuals. We think that the NAA decrease representing neuron loss in the LGB can be demonstrated by MRS in a larger patient series.

Cho is a metabolite providing important clinical data in spectroscopic trials. Total Cho consists of the sum of phosphorylcholine and glycerophosphorylcholine. Cho is involved in the metabolism of the cell membrane phospholipids. In the proliferation of the cell membrane, free Cho is generated by phosphatidylcholine destruction. Cho is liberated from myelin, cell membranes, and other complex brain lipids as a result of several pathological processes, and becomes visible in the MRS. It increases with tumours, multiple sclerosis, head trauma, strokes, hypoxia, brain death, radiation, inflammation, diabetes, liver and kidney failure, dialysis, osmotic events, and liver transplantation. In demyelinising occurrences in the brain especially, Cho prominently increases. It reduces with infections and hepatic encephalopathy. ${ }^{26-28}$ Although in our study, the $\mathrm{Cho} / \mathrm{Cr}$ in LGB region was higher in the patients with glaucoma than in normal cases, no statistically significant difference was found between the two groups.

Lactate is the final product of glycolysis and accumulates when the oxidative metabolism is not able to meet the energy requirement. Lactate peaks in MRS at 1.32 p.p.m.. It also makes a second peak at 4.1 p.p.m., but this peak is usually suppressed as it is near the water peak. Lactate is very low in a normal brain.

Lactate is present in the human vitreous at a higher concentration than in other tissues, likely reflecting substantial normal retinal aerobic and anaerobic glucose metabolism. ${ }^{29,30}$ Although its concentration is high in the vitreous, it is not high enough to be detected by the MRS. The proximity of the vitreous to the optic nerve and retina allows the diffusion of lactate into the vitreous. Elevated lactate concentration in the optic nerve and/or retina is generally reflected in the vitreous. ${ }^{23}$

First, Buchi and subsequently Joo et al ${ }^{31,32}$ studied microscopically the retinal ganglion cells that are about to die histopathologically in an experimental ischaemic glaucoma model developed by increasing the IOP of rabbits, and determined that some cells showed necrotic morphology, and some in apoptotic morphology.

Although the reason for ganglion cell death in glaucoma is hard to be explained by a single mechanism, many authors named this situation as cell death forms such as 'cell death secondary to necrosis', 'cell death between apoptosis and necrosis', 'sequential apoptosis/necrosis', or 'necrosis developed above apoptosis' ${ }^{33}$ The lactate peak observed in 11 of the patients with glaucoma, but not in the control group in our study suggests different and sophisticated mechanisms including necrotic cell death as well as apoptotic cell death.

Gupta et $a l^{34}$ studied bilateral LGB regions of 10 patients with glaucoma with the MRI method. They determined that LGB regions of the glaucoma patients became smaller in volume when compared with controls, and they concluded that atrophy occurred in the LGB regions of the patients with glaucoma. Weber et al ${ }^{35}$ in a study on monkeys with glaucoma experimentally created, found a decline in the neuronal volume and atrophy in the LGB region. Gupta et al ${ }^{36}$ identified degenerative changes neuroradiologically in the optic nerve, the LGB region and the visual cortex of a 79-yearold patient followed-up for glaucoma with visual field loss. Yucel et al ${ }^{37}$ demonstrated atrophy

histopathologically in magnocellular and parvocellular cell layers of the LGB regions of monkeys with experimentally formed glaucoma. All of these studies show that glaucoma leads to neuronal loss in the optic nerve and LGB region. It is a natural consequence that these changes occurring in LGB alter some metabolites. Our study has demonstrated that the MRS method reveals these changes.

In conclusion, we think that the MRS method, which is able to measure biochemical structure and metabolites of tissues non-invasively, may move the diagnosis of glaucoma one-step forward and open new horizons in the understanding and diagnosing of glaucoma at cellular level.

\section{Summary}

What was known before

- Only invasive procedures and cellular changes were known before.

What this study adds

- It adds a new method confirming the diagnosis of glaucoma and the role of apoptosis in etiopathogenesis of glaucoma.

\section{Conflict of interest}

The authors declare no conflict of interest.

\section{Acknowledgements}

This study was supported by the Inonu University Scientific Research Project and presented as a poster in ASCRS 2010 meeting, Boston, USA and awarded with the 'Special Interest' prize. 


\section{References}

1 Honkanen RA, Baruah S, Zimmerman MB, Khanna CL, Weaver YK, Narkiewicz J et al. Vitreous amino acid concentrations in patients with glaucoma undergoing vitrectomy. Arch Ophthalmol 2003; 121: 183-188.

2 Carter CJ, Brooks DE, Doyle DL, Drance SM. Investigations into a vascular etiology for low-tension glaucoma. Ophthalmology 1990; 97: 49-55.

3 Quigley HA, McKinnon SJ, Zack DJ, Pease ME, KerriganBaumrind LA, Kerrigan DF et al. Retrograde axonal transport of BDNF in retinal ganglion cells is blocked by acute IOP elevation in rats. Invest Ophthalmol Vis Sci 2000; 41: $3460-3466$

4 Casson RJ. Possible role of excitotoxicity in the pathogenesis of glaucoma. Clin Exp Ophthalmol 2006; 34: 54-63.

5 Okuno T, Oku H, Sugiyama T, Ikeda T. Glutamate level in optic nevre head is increased by artificial elevation of intraocular pressure in rabbits. Exp Eye Res 2006; 82: 465-470.

6 Marcaggi P, Hirji N, Attwell D. Release of L-aspartate by reversal of glutamate transporters. Neuropharmacology 2005; 49: 843-849.

7 Dreyer EB, Zurakowski D, Schumer RA, Podos SM, Lipton SA. Elevated glutamate levels in vitreous body of humans and monkeys with glaucoma. Arch Ophthalmol 1996; 114: 299-305.

8 Hare WA, Wheeler L. Experimental glutamatergic excitotoxicity in rabbit retinal ganlion cells: block by memantine. Invest Ophthalmol Vis Sci 2009; 50: 2940-2948.

9 Ju WK, Kim KY, Angert M, Duong-Polk KX, Lindsey JD, Ellisman $\mathrm{MH}$ et al. Memantine blocks mitochondrial OPA 1 and cytochrome c release and subsequent apoptotic cell death in glaucomatous retina. Invest Ophthalmol Vis Sci 2009; 50: 707-716.

10 Danesh-Meyer HV. Neuroprotection in glaucoma:recent and future directions. Curr Opin Ophthalmol 2011; 22: 78-86.

11 Osborne NN. Recent clinical findings with memantine should not mean that the idea of neuroprotection in glaucoma is abandoned. Acta Ophthalmol 2009; 87: 450-454.

12 Cello KE, Nelson-Quigg JM, Johnson CA. Frequency doubling technology perimetry for detection of glaucomatous visual field loss. Am J Ophthalmol 2000; 129: 314-322.

13 Johnson CA, Adams AJ, Casson EJ, Brandt JD. Progression of early glaucomatous visual field loss as detected by blueon-yellow and standard white on white automated perimetry. Arch Ophthalmol 1993; 111: 651-656.

14 Castillo M, Kwock L, Mukherji SK. Clinical applications of proton MR spectroscopy. Am J Neuroradiol 1996; 17: 1-15.

15 Kerr JF, Wyllie AH, Currie AR. Apoptosis: a basic biological phenomenon with wide-ranging implications in tissue kinetics. Br J Cancer 1972; 26: 239-257.

16 Bosman FT, Visser BC, Ooveren JV. Apoptosis: pathophysiology of programmed cell death. Path Res Pract 1996; 192: 676-683.

17 Arends MJ, Morris RG, Wyllie AH. Apoptosis, the role of endonuclease. Am J Pathol 1990; 136: 593-608.

18 Nickells RW. The molecular biology of retinal ganglion cell death: caveats and controversies. Brain Res Bull 2004; 62: 439-446.

19 Nickells RW. Apoptosis of retinal ganglion cells in glaucoma: an update of the molecular pathways involved in cell death. Surv Ophthalmol 1999; 43: 151-161.
20 Osborne NN, Chidlow G, Layton CJ, Wood JP, Casson RJ Melena J. Optic nerve and neuroprotection strategies. Eye 2004; 18: 1075-1084.

21 Yucel YH, Gupta N, Zhang Q, Mizisin AP, Kalichman MW, Weinreb RN. Memantine protects neurons from shrinkage in the lateral geniculate nucleus in experimental glaucoma. Arch Ophthalmol 2006; 124: 217-225.

22 Berkowitz BA, Bansal N, Wilson CA. Non-invasive measurement of steady-state vitreous lactate concentration. NMR Biomed 1994; 7: 263-268.

23 Rucker JC, Biousse V, Mao H, Sandbach J, Constantinidis I, Newman NJ. Detection of lactate in the human vitreous body using proton magnetic resonance spectroscopy. Arch Ophthalmol 2003; 121: 909-911.

24 Angelie E, Bonmartin A, Boudraa A. Regional differences and metabolic changes in normal aging of the human brain: proton MR spectroscopic imaging study. Am J Neuroradiol 2002; 22: 119-127.

25 Smith JK, Londono A, Castillo M, Kwock L. Proton magnetic resonance spectroscopy of brain-stem lesions. Neuroradiol 2002; 44: 825-829.

26 Alkan A, Kutlu R, Aslan M, Yakinci C. Adrenoleukodystrophy: single voxel MR spectroscopy findings (case report). Tani Girisim Radyol 2004; 10: 200-203.

27 Lin A, Ross BD, Harris K, Wong W. Efficacy of proton magnetic resonance spectroscopy in neurological diagnosis and neurotherapeutic decision making. NeuroRx 2005; 2: 197-214.

28 Kanamori M, Kumabe T, Shimizu H. (201)TI-SPECT, (1)H-MRS, and MIB-1 labeling index of central neurocytomas: three case reports. Acta Neurochir 2002; 144: 157-163.

29 Poitry-Yamate CL, Poitry S, Tsacopoulos M. Lactate released by Muller glial cells is metabolized by photoreceptors from mammalian retina. J Neurosci 1995; 15: 5179-5191.

30 Sebag J. The vitreous. In: Hart W ed. Adler's Physiology of the eye. 9th edn. Mosby-Year Book Inc: St Louis, Mo, 1992; 268-340.

31 Buchi ER. Cell death in the rat retina after a pressureinduced ischemia-reperfusion insult: an electron microscopic study, 1: ganglion cell layer and inner nuclear layer. Exp Eye Res 1992; 55: 605-613.

32 Joo CK, Choi JS, Ko HW, Park KY, Sohn S, Chun MH et al. Necrosis and apoptosis after retinal ischemia: involvement of NMDA- mediated excitotoxicity and p53. Invest Ophthalmol Vis Sci 1999; 40: 713-720.

33 Obsorne NN, Wood JP, Chidlow G, Bae JH, Melena J, Nash MS. Ganglion cell death in glaucoma: What do we really know? Br J Ophthalmol 1999; 83: 980-986.

34 Gupta N, Greenberg G, Tilly LN, Gray B, Polemidiotis M, Yucel $\mathrm{YH}$. Atrophy of the lateral geniculate nucleus in human glaucoma detected by magnetic resonance imaging. Br J Ophthalmol 2009; 93: 56-60.

35 Weber AJ, Chen H, Hubbard WC, Kaufman PL. Experimental glaucoma and cell size, density, and number in the primate lateral geniculate nucleus. Invest Ophthalmol Vis Sci 2000; 41: 1370-1379.

36 Gupta N, Ang LC, Tilly LN, Bidaisee L, Yucel YH. Human glaucoma and neural degeneration in intracranial optic nevre, lateral geniculate nucleus and visual cortex. $\mathrm{Br} J$ Ophthalmol 2006; 90: 674-678.

37 Yucel YH, Zhang Q, Weinreb RN, Kaufman PL, Gupta N. Atrophy of relay neurons in magno- and parvocellular layers in the lateral geniculate nucleus in experimental glaucoma. Invest Ophthalmol Vis Sci 2001; 42: 3216-3222. 